\title{
The COVID-19 Pandemic as a Stress Test - ensuring Individual Medical Respiratory Care: Aspects to Objectify the Discussion
}

\section{P. Kremeier (Peter Kremeier)}

Simulation Center for Clinical Ventilation, Karlsruhe, DE.

\section{E-mail address:}

pk@respicode.de

\section{Reprint address:}

Peter Kremeier

Simulation Center for Clinical Ventilation

Karlsruhe

Germany

Source: Clinical Social Work and Health Intervention

Volume: 12

Issue: 2

Pages: $63-67$

Cited references: 5

\section{Reviewers:}

Clauss Muss

I-GAP Zurich, $\mathrm{CH}$

Peter Marks

London, GB

\section{Keywords:}

Stress test. COVID-19. Individual Medical Respiratory.

\section{Publisher:}

International Society of Applied Preventive Medicine i-gap

CSWHI 2021; 12(2): 63 - 67; DOI: 10.22359/cswhi_12_2_12 (C) Clinical Social Work and Health Intervention

\section{Abstract:}

The COVID-19 pandemic confronts intensive care medicine with a new clinical picture, which is manifested in various forms and which clearly differs from the classic acute respiratory distress syndrome (ARDS). Ventilation therapy for COVID-19 pneumonia is complex and, contrary to previous guidelines for the treatment of acute respiratory failure, an increasing number of these patients do not primarily receive invasive ventilation. High-flow $\mathrm{O}_{2}$ therapy and non-invasive ventilation by mask or ventilation helmet have become key treatment options. In endeavours to provide respiratory care to all segments of the population whenever necessary, other therapeutic devices may be employed. The fact that milder cases of these diseases can also be treated with less expensive out-of-hospital ventilators and HFOT devices and that a full-fledged intensive care ventilator may not be imperative must be considered in the 
final decision. Nevertheless, answers to the triage and allocation of ventilators must be found in a discussion involving society as a whole and the health sciences in particular. The health sciences are called upon to contribute to the public debate on the distribution of all necessary resources during the pandemic.

\section{Introduction}

In November 2019, a previously unknown virus emerged in Wuhan, China, and has since spread across the globe. This global pandemic also reached Germany with its first detected case in Gauting on 27 January 2020. The SARS-CoV2 virus responsible for the current pandemic is the causative agent of severe acute respiratory syndrome, and the resulting disease is known as COVID-19. Every day during the first wave of the infection, the media reported on the challenges faced by the public health care system. Despite massive national and international efforts the shortage of ventilation capacity was a daily topic in the media and discussions among experts revolved around which intensive care patients should be ventilated and which should not (or no longer) be treated in intensive care. It was not only in Italy that the media reported cases in which patients were triaged to decide on who could not receive intensive care because of the insufficient availability of ventilators. In this emotional situation, creative minds from various branches of industry tried to develop crisis ventilators, the basic principle of which was mechanically filling and squeezing a type of bag-valve mask. In Germany, the already high number of beds for ventilator-dependent patients per 100,000 inhabitants was also significantly increased, but there were supply bottlenecks. It was therefore also considered likely that within a short period of time and despite the gradual capacity increases, ICU beds for ventilator-dependent patients would not be available in Germany for all those who needed them.

The health sciences are called upon to discuss diverse questions regarding the provision of resources for all population groups and to develop proposals for solutions. These encompass not only ventilation capacities, but also preventive measures such as protective equipment, vaccines and vaccination categories. To objectify the often purely emotional discussions, individual aspects in connection with ventilation medicine will be examined below.

\section{COVID-19 between an asymptomatic course and severe respiratory failure}

According to a situation report provided by the World Health Organization (WHO), SARS$\mathrm{CoV}-2$ has an overall mortality rate of $1.4 \%$, with documented rates varying from $<1 \%$ to $>7 \%$ depending on the demographic composition of the study population (1). However, the results of studies conducted around the world have shown that the actual number of infected cases is much higher than the cumulative number of confirmed infections, probably due to a lack of screening among asymptomatic or mildly symptomatic individuals (2). Due to its widespread symptomatology, multi-organ involvement and broad spectrum of disease severity, ranging from asymptomatic to symptomatic, mild or moderate to severe cases necessitating intensive care treatment to fatal outcomes of the disease, COVID-19 has caught medical practitioners worldwide unawares. According to the WHO, approximately $80 \%$ of COVID-19 infections are mild to moderate or asymptomatic; $15 \%$ develop severe symptoms requiring supplemental oxygen; and $5 \%$ experience a critical illness with complications such as respiratory failure, acute respiratory distress syndrome (ARDS), sepsis and septic shock, acute kidney injury, thromboembolism and/or multiple organ failure. Other acute and life-threatening conditions that have been described in COVID-19 patients include acute pulmonary embolism, acute coronary syndrome, delirium and acute stroke.

\section{Ventilation of COVID-19 patients with severe respiratory failure}

ARDS caused by COVID-19 pneumonia does not correspond to "conventional" ARDS [(3). Although ARDS is also associated with inflammatory activation, endothelial cell damage and hypercoagulability in the pulmonary system, as well as with increased dead space, right ventricular dysfunction and decreased pulmonary compliance, COVID-19-induced ARDS has some specific, pathophysiological characteris- 
tics. In COVID pneumonia, there is an extremely inhomogeneously distributed pattern of damage that does not show the gravity-dependent distribution of hyperinflated, functional and atelectatic lung tissue of conventional ARDS. (Google defines Atelectasis (at-uh-LEK-tuh-sis) as a complete or partial collapse of the entire lung or area (lobe) of the lung. It occurs when the tiny air sacs (alveoli) within the lung become deflated or possibly filled with alveolar fluid). Due to this inhomogeneous lung damage, shear forces - which can reach values many times higher than the inspiratory pressure - develop between atelectatic and hyperinflated lung areas. These regional shear forces can also negatively affect adjacent, healthy areas in the lung. As these shear forces are significantly increased by controlled ventilation, we suspect this to be one of the reasons why controlled ventilation often considerably worsens the pulmonary situation in patients with COVID-19-related ARDS. Marini et al. defined different stages of COVID-19 pneumonia with types $\mathrm{L}$ and $\mathrm{H}$, which differ substantially in their pathophysiology. While, despite severe hypoxia and interstitial edema, the compliance of the probably non-recruitable lung tissue is still high; type $\mathrm{H}$ is characterized by low compliance with a high degree of right-to-left shunting due to progressive damage to the lungs. This stage requires a higher PEEP (positive endexpiratory pressure). Ventilated patients have a markedly poor outcome with a high mortality rate of over $50 \%$ [4]. Currently, there are hardly any promising pharmacological therapies to effectively influence the course of the disease. It has been repeatedly observed that patients with COVID-19 pneumonia often develop a pathologically increased respiratory drive, which very likely leads to further damage to the lungs. The extent to which the occurrence of atelectasis with an extremely inhomogeneous distribution pattern is related to this pathological respiratory drive is unclear. We can assume that, due to inhomogeneous lung injury, a normal or increased tidal volume meets a significantly lower functional lung volume depending on the lung region and can therefore result in P-SILI (Patient SelfInflicted Lung Injury). Accordingly, at this level of severity of COVID-19, a full intensive care ventilator with all functions for lung-protective ventilation must be provided.

\section{Ventilation of COVID-19 patients with lower COVID-19 severity}

In addition to the 5\% of COVID-19 patients with severe respiratory failure, a further $15 \%$ develop severe disease requiring at least oxygen or milder methods of "ventilation" or respiratory therapy. Besides non-invasive ventilation by means of a mask or ventilation helmet, the socalled high-flow $\mathrm{O}_{2}$ therapy (HFOT) is of particular importance. These milder treatment strategies are not only available in intensive care ventilators, but can also be implemented with less expensive out-of-hospital ventilators and HFOT systems. In high-flow $\mathrm{O}_{2}$ therapy combined with a respiratory humidifier, for example, heated and humidified oxygen is applied at high flow rates of up to 70 liters per minute via a special nasal cannula. It is often possible to successfully oxygenate patients with this type of acute hypoxemic respiratory failure, and this form of therapy is usually very well accepted by the patient. If HFOT no longer suffices to provide adequate oxygenation and decarboxylation with tolerable breathing effort, then non-invasive ventilation (NIV) is the next best level of escalation. In this case it is possible to provide the patient with pressure support and, even more crucially, with positive end-expiratory pressure (PEEP). With the onset of the current pandemic, both high-flow $\mathrm{O}_{2}$ therapy and mask ventilation became highly controversial due to the potential hazard associated with aerosol formation for the health care personnel. The spectrum ranged from the complete abandonment of HFOT and NIV (with early intubation) to the generous use of NIV (in some cases even on normal wards). First studies have shown that the increased aerosol formation of these procedures can be reduced to an acceptable level by appropriate protective measures such as filter systems and masks for medical staff.

\section{Patient's will versus indication for ventilation}

Particularly when resources are scarce, medical treatment teams must be provided with socially and ethically supported decision-making aids. When questioning the use of ventilation therapy, not only the number of available ventilators, but ultimately the overall situation must be assessed with the patient at the center of deliberations. In addition to the medical, legal and political discussions and in addition to the ethics 
Figure 1: modified according to the $S 1$ guideline „Decisions on the allocation of intensive care resources in the context of the COVID-19 pandemic". AWMF [The Association of the Scientific Medical Societies in Germany] registration number 040-013 dated 17.04.2020.

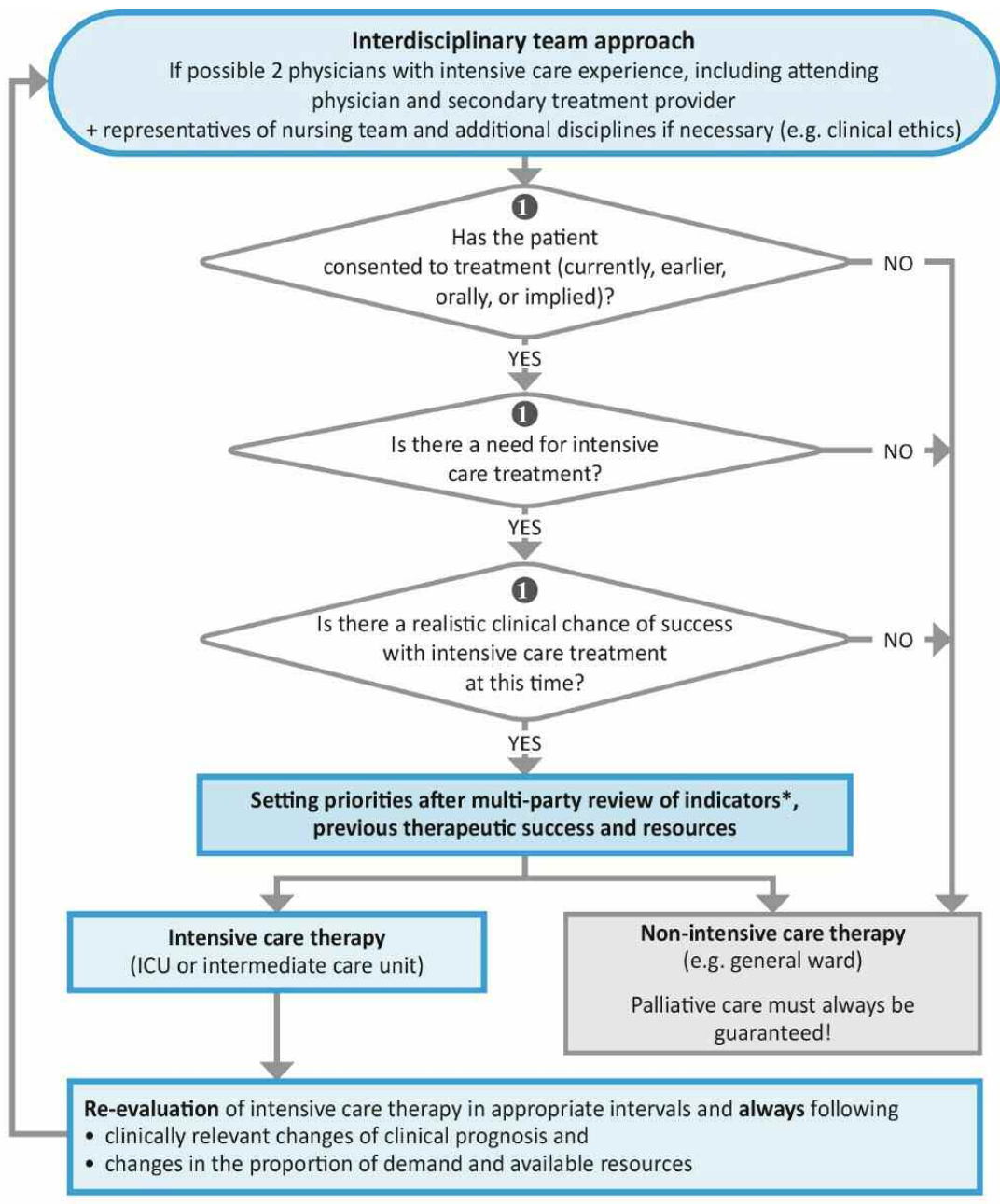

\begin{tabular}{|c|c|}
\hline \multicolumn{2}{|c|}{ Indicators of poor prognosis in initial assessment or re-evaluation } \\
\hline $\begin{array}{l}\text { Current condition } \\
\text { - Higher severity (e.g. acute respiratory distress } \\
\text { syndrome [ARDS]) } \\
\text { - Accompanying acute organ failure (e.g. SOFA } \\
\text { score > 11) } \\
\text { - If applicable, prognostic markers for COVID-19 } \\
\text { patients }\end{array}$ & $\begin{array}{l}\text { Comorbidities } \\
\text { Individual severe comorbidity with significant } \\
\text { impact on long-term prognosis: } \\
\text { - Chronic organ failure } \\
\text { - End-stage organ dysfunction } \\
\text { - Advanced neurological disease } \\
\text { - Advanced oncological disease }\end{array}$ \\
\hline $\begin{array}{l}\text { General health condition } \\
\text { - Advanced frailty (e.g. Clinical Frailty Scale, CFS) }\end{array}$ & $\begin{array}{l}\text { - Severe immune deficiency } \\
\text { - Multi-morbidity }\end{array}$ \\
\hline
\end{tabular}

(1) Prequisites for terminating intensive care therapy after re-evaluation
- Continuing the intensive care therapy runs counter to the patient's (declared,
presumed) will
- Therapeutic goal is no longer realistically achievable
- Treatment attempts have been unsuccessful after an observation period with
previously defined criteria
- Progressive multiple organ failure (e.g. significant increase of SOFA score [>2]
within 24 hours)


committees, the health sciences must also deal with questions of ensuring adequate health care for the individual and the need for a quota system for ICU beds and beds for ventilator-dependent patients.

The COVID-19 pandemic confronts intensive care medicine with a new clinical picture, which is manifested in various forms and which clearly differs from the classic acute respiratory distress syndrome (ARDS). Ventilation therapy for COVID-19 pneumonia is complex and, contrary to previous guidelines for the treatment of acute respiratory failure, an increasing number of these patients do not primarily receive invasive ventilation. High-flow $\mathrm{O}_{2}$ therapy and non-invasive ventilation by mask or ventilation helmet have become key treatment options. In endeavors to provide respiratory care to all segments of the population whenever necessary, other therapeutic devices may be employed. The fact that milder cases

of these diseases can also be treated with less expensive out-of-hospital ventilators and HFOT devices and that a full-fledged intensive care ventilator may not be imperative must be considered in the final decision. Nevertheless, answers to the triage and allocation of ventilators must be found in a discussion involving society as a whole and the health sciences in particular. The health sciences are called upon to contribute to the public debate on the distribution of all necessary resources during the pandemic.

\section{References}

1. WORLD HEALTH ORGANIZATION (2020) Clinical Management of COVID-19. Interim guidance. 27. Mai 2020.

2. POLLAN M, PEREZ-GOMEZ B, PASTORBARRIUSO R, et al. (2020) Prevalence of SARS-CoV-2 in Spain (ENE-COVID): a nationwide, population-based seroepidemiological study [published online ahead of print, 2020 Jul 3]. Lancet. pp 396:535-44. pii: S0140-6736(20)31483-5.

3. MARINI JJ, DELLINGER RP, BRODIE D (2020) Integrating the evidence: confronting the COVID-19 elephant. Intensive Care Med. 46: pp 1904-7. doi:10.1007/s00134-02006195-z.

4. KARAGIANNIDIS C, MOSTERT C, HENTSCHKER C, VOSHAAR T, MAL-
ZAHN J, SCHILLINGER G, et al. (2020)

Case characteristics, resource use, and outcomes of 10021 patients with COVID-19 admitted to 920 German hospitals: an observational study. The Lancet Respiratory Medicine. 8: pp 853-62.

5. BROCHARD L, SLUTSKY A, PESENTI A (2017) Mechanical Ventilation to Minimize Progression of Lung Injury in Acute Respiratory Failure. Am J Respir Crit Care Med. pp 195:438-42. doi:10.1164/rccm.201605$1081 \mathrm{CP}$. Further literature sources from the author. 